\title{
Simulation of Two Traffic Options on a Heavily Traveled Road Adjacent to Los Alamos National Laboratory
}

\author{
Ed Van Eeckhout, Ph.D., P.E. \\ LaRon Smith, Ph.D. \\ Kriste Henson, M.S. \\ Los Alamos National Laboratory, Los Alamos, NM 87545
}

\begin{abstract}
The TRansportation ANalysis SIMulation System (TRANSIMS) team at Los Alamos National Laboratory evaluated the proposed conversion of Diamond Drive on Laboratory property from four to three lanes. This conversion was meant to address safety issues caused by left turns from Diamond Drive onto two crossing streets (visibility problems for oncoming traffic) and the lane widths being non-standard (too narrow). Widening the road was not considered an option because of the expected high cost.
\end{abstract}

For this analysis, the following situations were evaluated:

- A 4-lane versus 3-lane road;

- Morning and evening peak traffic;

- Merging north versus merging south of the largest intersection for the proposed 3lane case, morning peak; and

- Potential traffic flux uncertainties of up to $20 \%$ in the morning peak traffic.

The results were quite dependent on the simulated signal timings, and can be summarized as follows:

- In terms of travel time through the system, the 3-lane option would work, on the average, about the same as the 4-lane situation;

- A few travelers would experience a $25-50 \%$ increase in travel time over the average travel time of $3.5-4$ minutes;

- The 3-lane option will place the morning traffic at close to its maximum throughput. Additional traffic volumes would be expected to degrade the system; and

- There is a distinct, dramatic advantage to merging south rather than north of the Diamond/Jemez intersection in the 3-lane option.

\section{TRANSIMS Simulations}

The TRANSIMS microsimulation uses a cellular automata (CA) approach to traffic simulation. The network lanes are partitioned into 7.5-meter cells. A cell may or may not contain a vehicle. The vehicle positions are updated every second based on a few simple rules that have been shown to produce a reasonable representation of traffic. Complete documentation of the TRANSIMS microsimulation and other modules can be found on the TRANSIMS web site: http://transims.tsasa.lanl.gov. The site also contains additional papers and documents describing the theoretical development and basis for the microsimulation methods. 
For this study, the roadway speed limits were all set at $15.65 \mathrm{~m} / \mathrm{s}(35 \mathrm{mph})$, which TRANSIMS converts to two cells as the maximum number of cells that a vehicle may move each second. While this is close to the $15.65 \mathrm{~m} / \mathrm{s}$, we used a vehicle slowing probability in the CA method that reduced the vehicle average velocity to approximately $14.4 \mathrm{~m} / \mathrm{s}$ in the absence of other vehicles, signals, or traffic signs. However, if no other vehicle is present in the next two consecutive cells, the majority of vehicles will accelerate to two cells per second $(15.65 \mathrm{~m} / \mathrm{s})$ in two seconds.

\section{The Diamond Drive Network}

Figure 1 shows the network for this study. We created the network by overlaying roads and parking lots on a satellite orthophoto taken in June 2000. This network represents the roads that vehicles travel across in the simulation. In addition, we specified the lanes on each road and the allowed turning movements through all intersections. Los Alamos' Facilities group provided drawings with specific lane configurations. Additionally, there are three locations where traffic must obey timing constraints. Other intersections have either stop/yield signs or no traffic control. Because the study's primary purpose was to understand the effect of Diamond Drive alternatives on Laboratory property, we did not model road features and intersections beyond the adjacent Diamond Drive feeders. Thus interactions with other Laboratory or Los Alamos County roadway signals or alternatives were not part of this study.

We created twelve "parking lots" where vehicles would begin and end their trips, located at the end of each potential route (Figure 1). Normally, TRANSIMS uses "activity" locations as places where travelers start and end various tasks. But for the purpose of this particular study, that TRANSIMS feature was not used. Instead, we began with the travelers and their associated vehicles (one traveler per vehicle) at each parking lot. For the 4-lane configuration, we modeled two lanes in each direction for the full length of Diamond Drive from Jemez Road to Pajarito Road. Appropriate turn pocket lanes were included at the Jemez Road intersection. For the 3-lane configuration, we modeled a single lane in each direction on Diamond Drive and added left turn pockets of length approximately $49 \mathrm{~m}$ at the Eniwetok and Sigma intersections and at the intersections for "parking lots" 4 and 5. A merge distance of length $123 \mathrm{~m}$ was included on the south side of the Jemez Road intersection to allow the two southbound lanes to merge to one. We also increased the northbound lanes from one to two lanes $123 \mathrm{~m}$ south of the Jemez Road intersection. For the case where the southbound merge would occur north of the Jemez Road intersection, we forced the right lane traffic crossing the Los Alamos Canyon Bridge to turn right onto West Jemez Road whereas the left lane traffic would continue straight. This is approximately $128 \mathrm{~m}$ before the Jemez Road intersection. Traffic going to East Jemez Road would then use the left turn pocket on the roadway segment from the bridge to the intersection. 


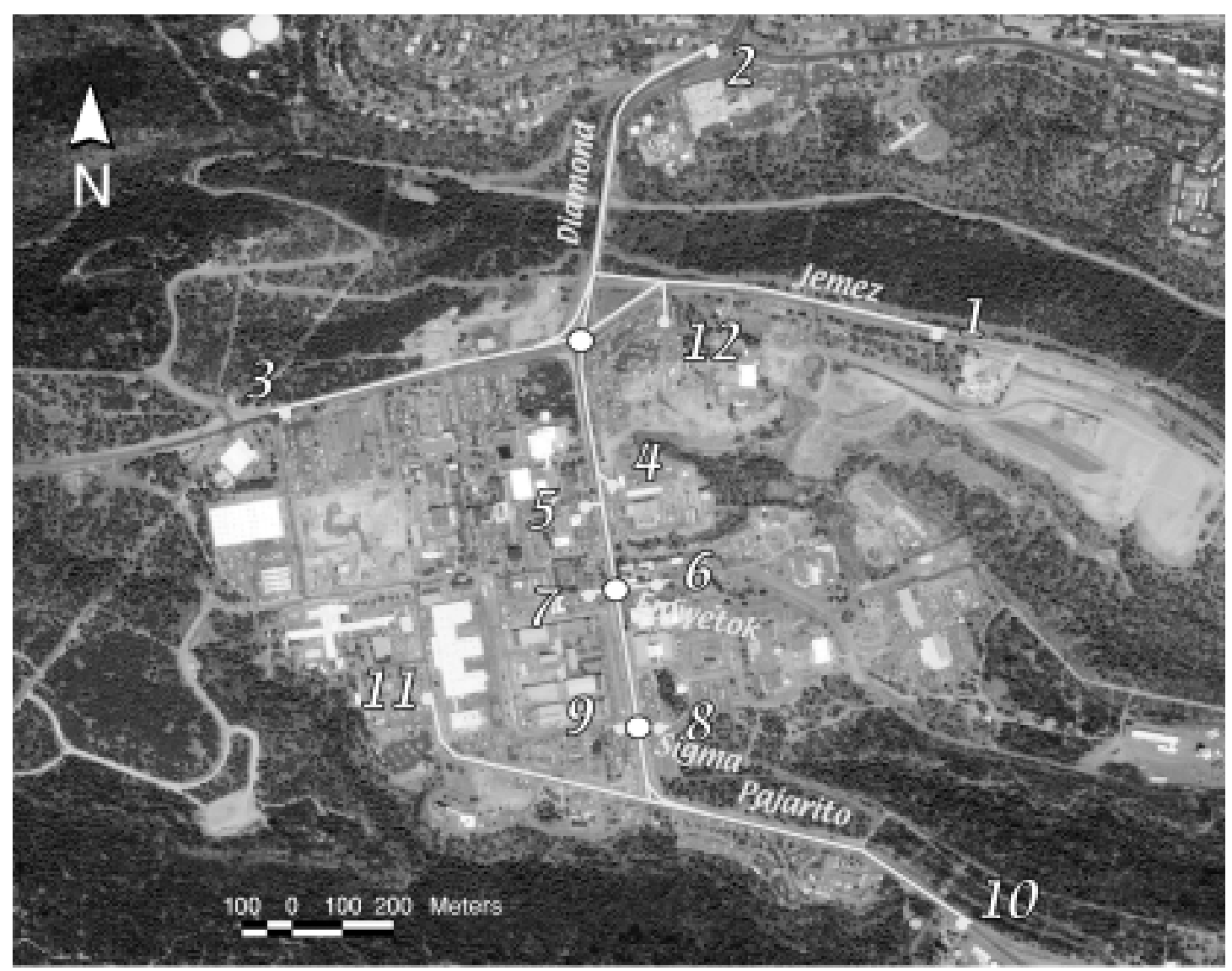

Figure 1. Network overlay on background satellite image of main laboratory area.

\section{Traffic Signals}

We modeled traffic signals at the Diamond Drive intersections with Jemez Road, Eniwetok Drive, and Sigma Drive (shown as white circles in Figure 1). Although these signals are actuated, we modeled them as pre-timed since it was assumed that the signals utilize maximum phase times during peak traffic volumes. We experimented with different signal cycle lengths and phase splits as we attempted to baseline the existing conditions. These results indicated a high sensitivity to the signal cycle lengths and phasings.

\section{Traffic Volumes}

TRANSIMS requires traveler plans (specific routes) and vehicle information as input for the microsimulation. For this simple network, we created traveler origindestination tables to match turn counts obtained during the month of December 1999 for the intersections in question. The number of trips from the origin parking lot to the destination parking lot is shown in Table 1 for the period 7 to $8 \mathrm{am}$. We did not simulate travelers for which the origin-destination value in the tables was less than five; in total, 3799 morning vehicles and 2928 afternoon vehicles were simulated.

Table 1. Trips from one parking lot to another from 7 am to 8 am in December 1999. 


\section{To From Parking Lot}

$\begin{array}{lllllllllllll} & \mathbf{1} & \mathbf{2} & \mathbf{3} & \mathbf{4} & \mathbf{5} & \mathbf{6} & \mathbf{7} & \mathbf{8} & \mathbf{9} & \mathbf{1 0} & \mathbf{1 1} & \text { Total } \\ \mathbf{1} & \mathbf{x} & 189 & 44 & 0 & 0 & 2 & 1 & 1 & 1 & 20 & 1 & 258 \\ \mathbf{2} & 161 & \mathbf{x} & 161 & 0 & 0 & 25 & 8 & 8 & 7 & \mathbf{2 2 3} & 13 & 606 \\ \mathbf{3} & 525 & 587 & \mathbf{x} & 0 & 0 & 14 & 4 & 4 & 4 & \mathbf{1 2 4} & 7 & 1270 \\ \mathbf{4} & 8 & 15 & 1 & \mathbf{x} & 0 & 0 & 0 & 0 & 0 & 6 & 0 & 29 \\ \mathbf{5} & 23 & 44 & 2 & 0 & \mathbf{x} & 0 & 0 & 0 & 0 & 17 & 1 & 88 \\ \mathbf{6} & 44 & 83 & 4 & 0 & 0 & \mathbf{x} & 0 & 0 & 0 & 27 & 2 & 161 \\ \mathbf{7} & 12 & 23 & 1 & 0 & 0 & 0 & \mathbf{x} & 0 & 0 & 42 & 2 & 81 \\ \mathbf{8} & 23 & 43 & 2 & 0 & 0 & 0 & 0 & \mathbf{x} & 0 & 26 & 2 & 96 \\ \mathbf{9} & 36 & 67 & 4 & 0 & 0 & 0 & 0 & 0 & \mathbf{x} & 19 & 1 & 126 \\ \mathbf{1 0} & \mathbf{2 0 1} & \mathbf{3 7 6} & 20 & 0 & 0 & 13 & 9 & 10 & 15 & \mathbf{x} & 120 & 764 \\ \mathbf{1 1} & 28 & 53 & 3 & 0 & 0 & 2 & 1 & 1 & 2 & 265 & \mathbf{x} & 356 \\ \text { Total } 1062 & 1480 & 243 & 0 & 0 & 56 & 23 & 24 & 29 & 768 & 150 & 3835\end{array}$

For most study analyses, we generated the traveler start times randomly based on a uniform distribution. And because our network did not extend to the signals external to the network (beyond the parking locations), we also did not generate any vehicle "platoon" effects or traffic surges coming from North Diamond.

The most significant traffic volumes between the origins and destinations are highlighted in the tables. Although we collected information on every traveler, this study concentrated on the performance of vehicles taking the most heavily traveled paths that extended the full length of Diamond Drive from the Jemez Road intersection to Pajarito Road in either direction. For the morning traffic, we were interested in the vehicles traveling from East Jemez and North Diamond to East Pajarito (paths 1 to 10 and 2 to 10, respectively) and from East Pajarito to North Diamond and West Jemez (paths 10 to 2 and 10 to 3, respectively). [Note that these location descriptions are relative to the simulation network and not necessarily part of the real road name.]

\section{3-Lane vs. 4-Lane Road, 140-Second vs. 120-Second Signal Timings}

For three simulations we compared the traveler travel times for the four paths. The results for traveling from Northern Diamond to East Pajarito (path 2 to 10), as shown in figure 3 , are representative of all network travel times. 


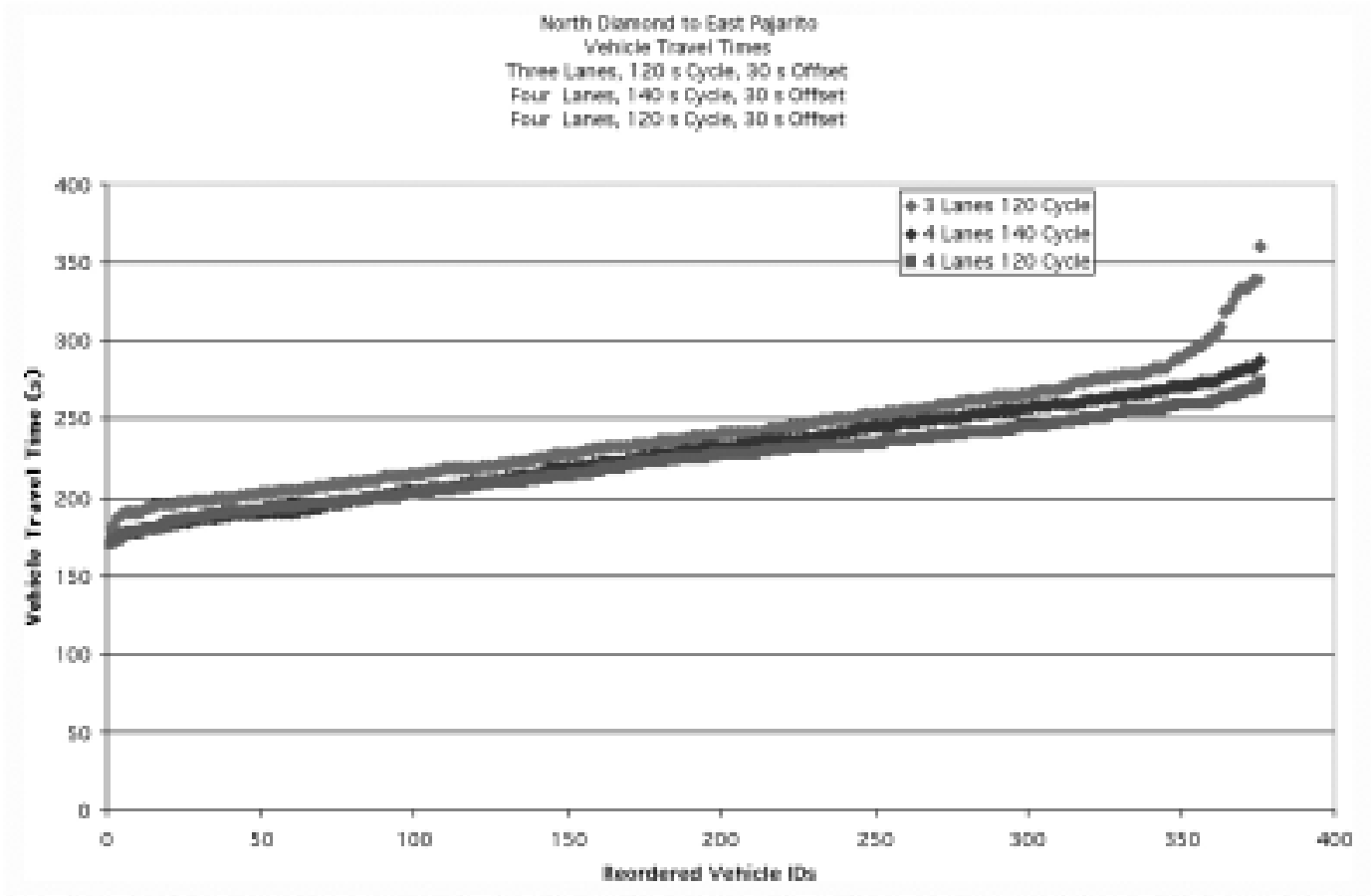

Figure 3. Comparison of North-Diamond-to-East-Pajarito (parking location 2 to 10) travel time distributions for 3-lane 120-second cycle, 4-lane 140-second cycle, and 4lane 120 -second cycle configurations.

For the 4-lane configuration, travel times during the120-second cycle signal configuration show a slight improvement over the current 140 -second cycle signal times. But on average, the 3-lane configuration does not differ much from the 4-lane configuration, although there are some travelers on the tail of the distribution whose travel times are $25-50 \%$ greater than the average travel time.

\section{3-Lane Configuration with Merge North of the Jemez Road Intersection}

Another alternative was whether it would be better to merge from two southbound lanes to one southbound lane before the Diamond/Jemez intersection. For this case, we forced the right lane traffic crossing the Los Alamos Canyon Bridge to turn right onto West Jemez Road whereas the left lane traffic would continue straight. This occurs approximately $128 \mathrm{~m}$ before the Jemez Road intersection. Traffic going to East Jemez Road would use the left turn pocket on the roadway segment from the bridge to the intersection.

Figure 4 compares the travel time distribution for the travelers from North Diamond to East Pajarito for the 3-lane northside and southside merge and for the current 4lane configuration. For the 3-lane northside merge, numerous travelers on this path have extremely long travel times ranging from 10 to 15 minutes. Southbound vehicles crossing the Los Alamos Canyon Bridge form a queue that backs up to the North Diamond "parking location." 

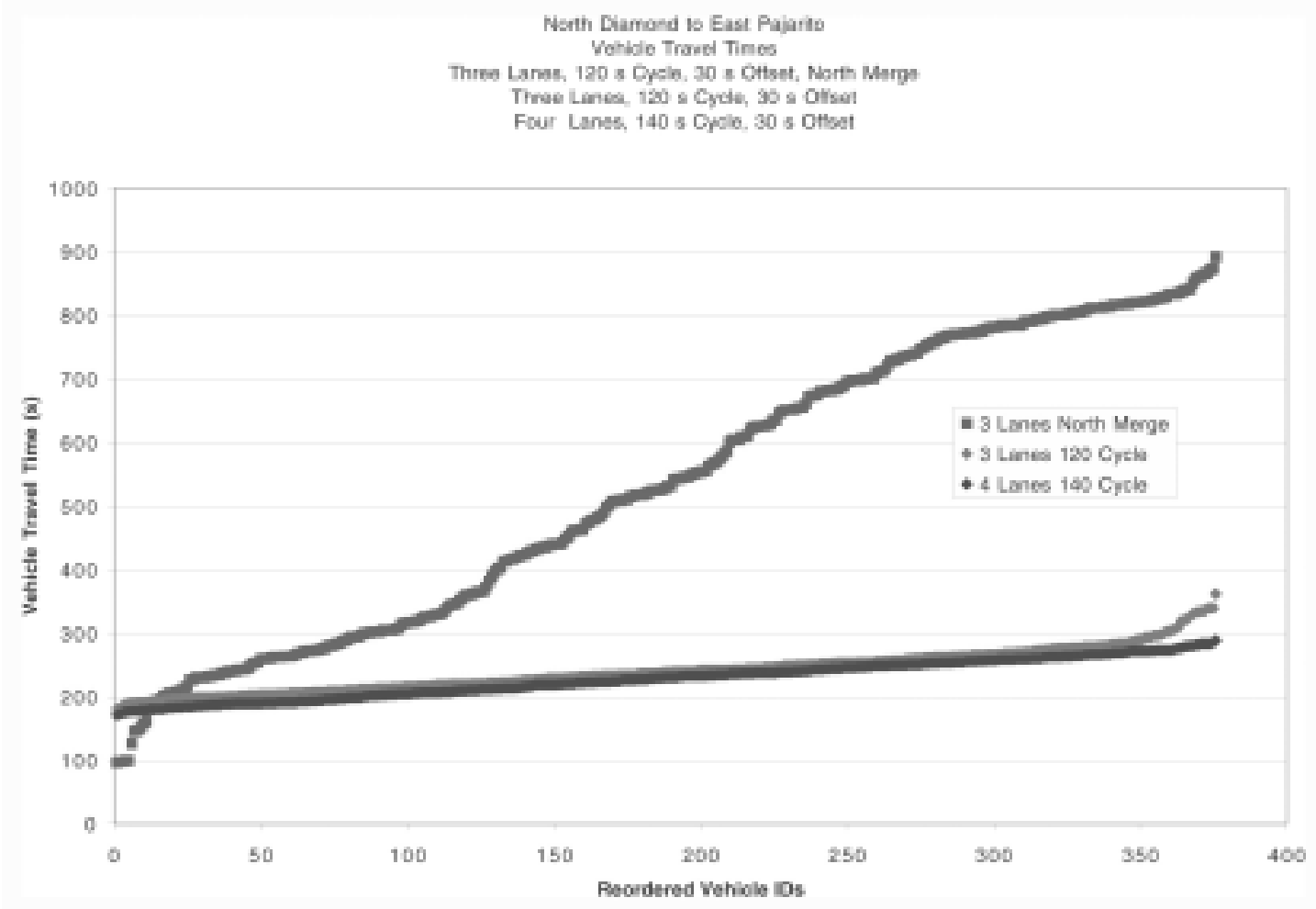

Figure 4. Travel time distributions for the North Diamond-to-East-Pajarito path (parking location 2 to 10) 3-lane configuration merging north of the Jemez Road intersection versus the 3-lane and 4-lane configuration merging south of the Jemez Road intersection.

The other paths are mostly unaffected by having the merge on the north side of the Jemez Road intersection.

\section{Increased Traffic Volumes}

Because of uncertainties in the turn counts used to derive the origin-destination tables, we were concerned about the performance of the alternatives if there were heavier traffic flow. To test this, we simulated $20 \%$ more vehicles during the morning peak period on both the 4-lane configuration and the proposed 3-lane configuration.

Figure 5 indicates that the 4-lane configuration handles the increased traffic volumes well, but that travelers on the 3-lane configuration would average over 50 seconds in increased travel time and a significant number of travelers would take over six minutes to get from North Diamond to East Pajarito.

\section{Discussion}

While TRANSIMS was created for regional planning studies, this is an application of the software to a local engineering study. We did not calibrate the microsimulation performance to real traffic data taken on Diamond Drive, although calibrations completed in other studies indicate that statistically the CA does a fairly good job of representing real traffic. Our confidence in the results was strengthened after we baselined the existing 4-lane configuration using current signal timings. While there 
may be some questions about the absolute validity of the individual vehicle results, we believe that, when comparing the alternatives in this study, the TRANSIMS results provide a reasonable indication of what can be expected.
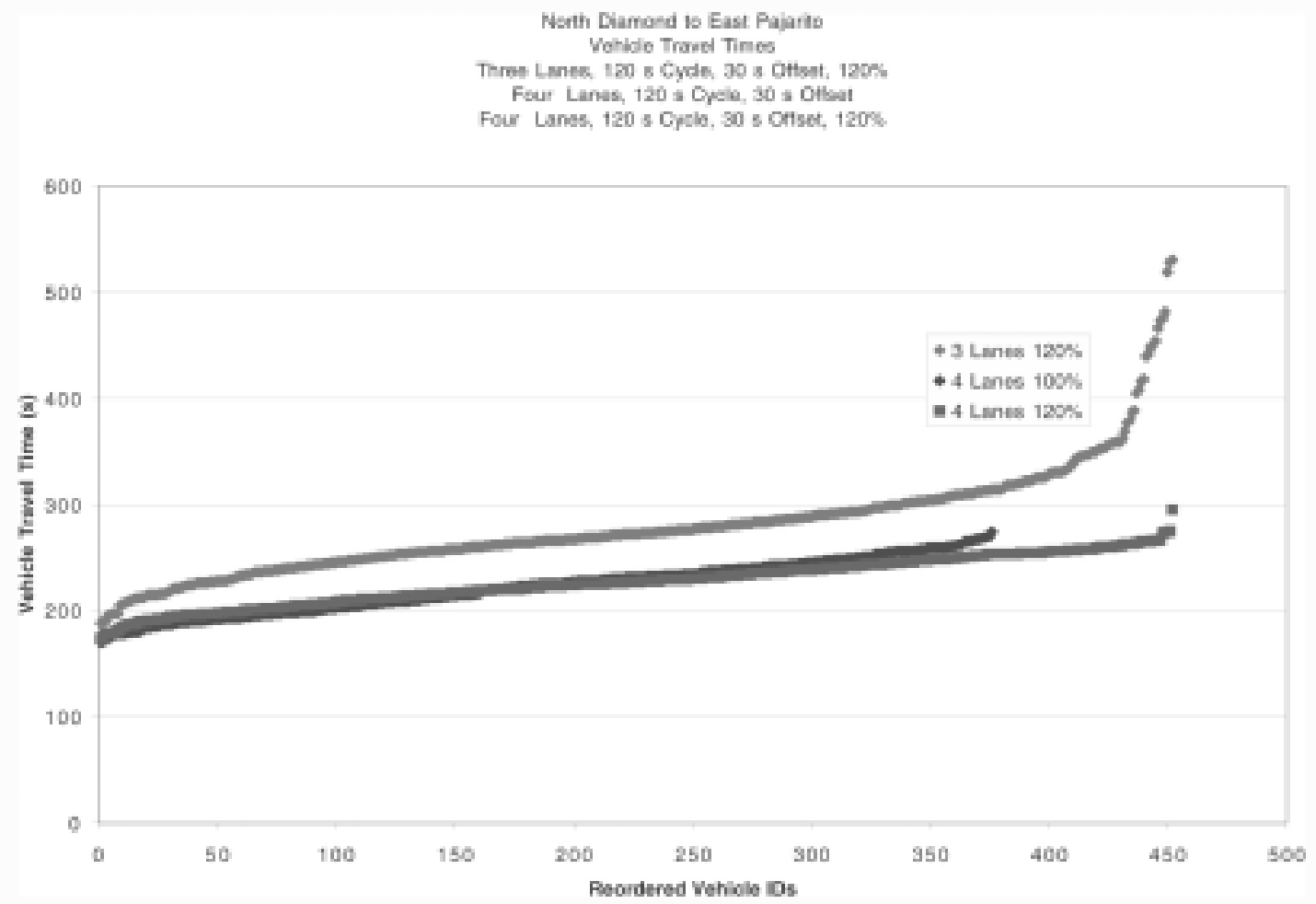

Figure 5. Comparison of time through the network (parking location 2 to 10 ) for $20 \%$ greater traffic in the 3-lane vs. 4-lane situation.

A brief examination of the morning origin-destination table (Table 1) and the capacities of the roadways feeding southbound Diamond Drive at the Jemez Road intersection indicates where problems might be expected after converting Diamond Drive to three lanes. In essence, the conversion to three lanes requires that all southbound traffic merge immediately south of the Jemez Road intersection, whereas currently only the through traffic to East Pajarito Road (53\% of the total) is required to merge to a single lane at the south end of Diamond Drive. Along the way, the other $47 \%$ of vehicles turn onto a side street. Of course, there is considerable maneuvering as vehicles attempt to move into proper lanes for turns or slip past vehicles that are turning.

There are some helpful factors to switching from 4-lane to 3-lane traffic. First, traffic from West Jemez onto southbound Diamond Drive is light, accounting for only about $3 \%$ of the southbound traffic. Second, about a third of the traffic originating from East Jemez is already moving as a single lane when it enters southbound Diamond. Furthermore, the Diamond-Jemez intersection signal prevents North Diamond traffic from converging onto southbound Diamond simultaneously with East Jemez traffic. There should be no significant delays caused by the merge to a single lane except for 
possible traffic backup from the Eniwetok. The signal phases at Jemez Road allow traffic from West Jemez Road to move prior to the East Jemez Road traffic; this should provide some opportunity to clear up residual merging from North Diamond. Also proper signal phasing at Eniwetok and Sigma should mitigate the backup. The TRANSIMS simulations indicate that there is a slight increase (approximately 10 seconds) in travel time from East Jemez to East Pajarito and about the same spread in travel time distribution when compared with the current 4-lane configuration.

The primary source of potential problems is the merging of the two southbound Diamond Drive lanes. Sixty-three percent (about 700 vehicles per hour) of the southbound traffic comes from North Diamond and must funnel in an orderly manner from two lanes to one lane. The TRANSIMS simulations indicate that it is possible to do this with only a slight penalty-a 12-second increase in average time to traverse from North Diamond to East Pajarito. However, the TRANSIMS simulations also indicate that about $20 \%$ of the Diamond Drive drivers on any given day may incur possibly 20-60 seconds additional delay above the average, and 5\% of the drivers may be delayed by more than a minute.

TRANSIMS simulations show that merging north of the Diamond-Jemez intersection would most assuredly be problematic.

The simulations of higher traffic volumes and different starting time distributions indicate that the three-lane configuration would be operating close to capacity during the morning peak. Perturbations from the baseline volume and uniform distribution are likely to increase southbound travel times for some travelers. The magnitude of the increase is dependent on the perturbation, but some instances could add more than a minute for most travelers and double the usual travel time for some travelers. We did not simulate the effect of minor incidents or traffic accidents.

The primary delays to northbound afternoon flow are the signals at Eniwetok and Sigma, but the TRANSIMS simulations indicate that there is little difference between the 3-lane and 4-lane configurations. The TRANSIMS simulations also indicate that the travel times for traffic from Eniwetok and Sigma onto Diamond differ little between the two configurations.

In summary, the TRANSIMS simulations show that converting Diamond Drive to three lanes on Laboratory property normally should not be burdensome to travelers although some will experience slightly longer travel times than for the current 4-lane configuration. However, during the morning peak traffic, the 3-lane configuration will be operating near capacity, and slight perturbations could cause significant traffic delays. 